\title{
Sensor-based M2M Agriculture Monitoring Systems for Developing Countries: State and Challenges
}

\author{
Lutful Karim \\ School of ICT, Seneca College of Applied Arts and Technology, Toronto, Canada \\ Email: lutful.karim@ senecacollege.ca
}

Alagan Anpalagan

Department of Electrical and Computer Engineering, Ryerson University, Toronto, Canada Email: alagan@ee.ryerson.ca

\begin{abstract}
Nidal Nasser
Electrical \& Computer Engineering Dept, College of Engineering, Alfaisal University, Saudi Arabia

Email: nnasser@alfaisal.edu

Jalal Almhana

Dept. of Computer Science, University of Moncton, Canada

Email: jalal.almhana@umoncton.ca
\end{abstract}

Received: May 31, $2013 \quad$ Accepted: October 25, $2013 \quad$ Published: October 31, 2013

DOI: $10.5296 /$ npa.v5i3. 3787

URL: http://dx.doi.org/10.5296/npa. v5i3. 3787

\begin{abstract}
Machine to machine (M2M) communication networks consist of thousands of low cost, low energy, low computational power and memory sensors nodes. Due to the autonomous monitoring, self organization, low power consumptions and remote accessibility of sensors, M2M communication networks gain momentum in environmental monitoring, pollution detection, agriculture, disasters monitoring and many similar applications. It is evident that
\end{abstract}


sensor-based agriculture monitoring systems are being designed and implemented mostly in the context of developed countries. However, these monitoring systems do not focus on the implementation of sensor-based M2M networks for the benefit of farmers in developing countries. This paper presents (i) the current state of the art research in sensor-based M2M communication networks for agriculture monitoring, (ii) several existing agricultural monitoring systems and compare them on different design factors, (iii) the technical framework of some recent deployment of agriculture monitoring systems in developing countries and their design challenges and (iv) major design and implementation differences of these monitoring systems in developed and developing countries.

Keywords: M2M Communications, Wireless Sensor Network, Agriculture Monitoring, Irrigation, Routing Protocol.

\section{Introduction}

Most countries depend on agriculture and hence, the optimal usage of land and water resources is very critical. Previous agriculture systems were labor dependent. The agriculture systems in developing countries are still labor dependent and do not use any crop management, pest/disease control or quality management systems. In addition, the traditional irrigation systems in agriculture use uniform water distribution in fields which is not optimal $[1,2]$. Hence, a technology based agricultural monitoring system is greatly required for farmers in developing countries.

It is estimated that $87 \%$ world's fresh water is consumed for irrigation and $33 \%$ of world's produced food uses irrigation. Hence, per capita fresh water is declining. On the other hand, global warming increases irrigation by $26 \%$ to maintain the world's current food production. Soil nutrients are also decreasing in the agricultural fields of the developing countries and so, the production decreases. Hence, a technology based agricultural monitoring system is greatly required.

Machine to machine (M2M) communication [3] is an emerging technological framework where daily use machines such as refrigerator, micro-oven, cell phone, laptop, tablet, smart phone, electric meter can communicate with each other and send data to the M2M central server or cloud through M2M area networks (e.g., sensor, RFID, Bluetooth) and core networks (e.g., WLAN, 3GPP, IP, WiMAX). Thus, monitoring devices and applications are connected to each other to work as a large scale framework of M2M. Figure 1 illustrates M2M communication network architecture. A machine is known as a machine type communication (MTC) device. The MTC devices communicate with each other and send data to the MTC gateway of M2M area networks through multi-hop communications. The MTC gateway again transmits data to the backhaul core networks through MTC routers having a large communication range. Hundreds of low cost, energy and computational power wireless sensor nodes are mostly used as MTC devices in the M2M area networks since sensors can be deployed easily, controlled automatically and monitored remotely. Moreover, 


\section{Macrothink}

sensors include low power radio and power management mechanisms to conserve energy for a longer time network operation. Hence, sensors are used as the integral part of M2M communication networks for different monitoring applications especially, agricultural monitoring. Agriculture monitoring systems mainly focus on monitoring the growth of crops, irrigation systems, monitoring and control of animals. Recently, many sensor-based agriculture monitoring systems have been designed and implemented (mostly for developed countries). However, only a few agricultural monitoring systems based on M2M communication networks exist in the literature such as agent-based M2M agricultural decision support system [4], and crop growth model [8]. Moreover, these systems do not consider using sensor-based M2M networks in the agriculture systems of developing countries $[1,5]$.

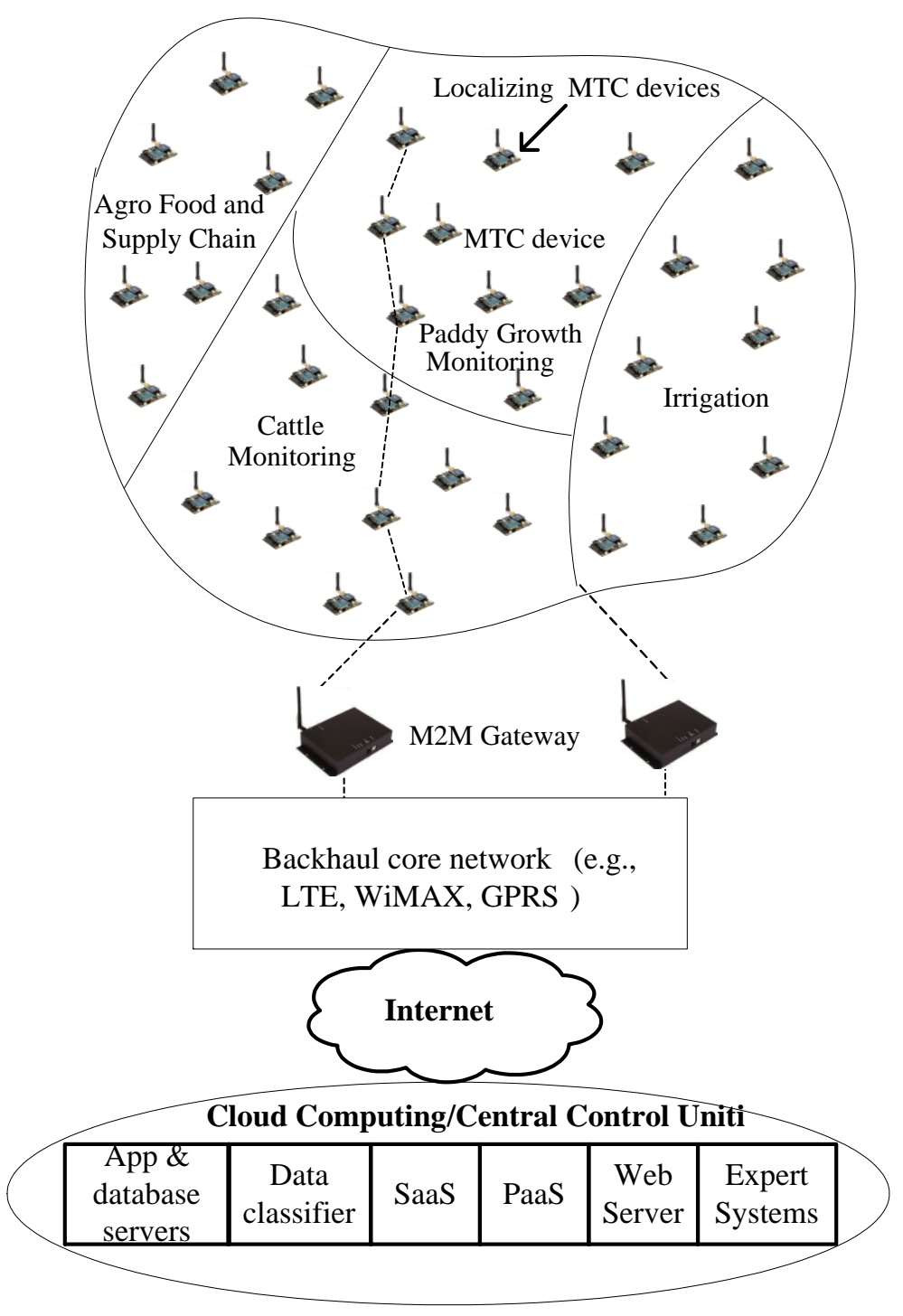

Figure 1. Sensor-based M2M communication networks. 
In this paper, we present several sensor-based M2M agriculture monitoring systems, identify important design criteria (e.g., topology and routing), challenges of designing sensor-based M2M agriculture monitoring systems for developing countries. We also identify the differences between agriculture monitoring systems of developed and developing countries.

The remainder of this paper is organized as follows. Section 2 presents several existing sensor-based agriculture monitoring systems with their architecture, working principle and limitations. In Section 3, we present design challenges of sensor-based M2M agriculture monitoring system in developing countries and major differences in the agricultural monitoring systems between developed and developing countries. Finally, Section 4 concludes this paper with some future research directions.

\section{Sensor-based M2M Agriculture Monitoring Systems}

This section describes different types of sensor-based agriculture monitoring systems. These monitoring systems are mainly designed to use in agriculture of developed countries.

\subsection{Paddy Growth Monitoring}

Rice is the main food for major population in the world. Thus, the production of rice should be increased to meet the demand of increased population. From statistics, it is found that farmers can produce rice/paddy 3-5 ton/hectare without technology while it is 5-10 tons with technology supports. However, farmers can neither manage cultivation techniques nor get technical support in rural areas from governments (mainly in developing region). On the other hand, labors in agriculture are in shortage now though the current agriculture systems in developing region require high labor power for water management and monitoring weather, soil condition, quality and nutrient of the plants.

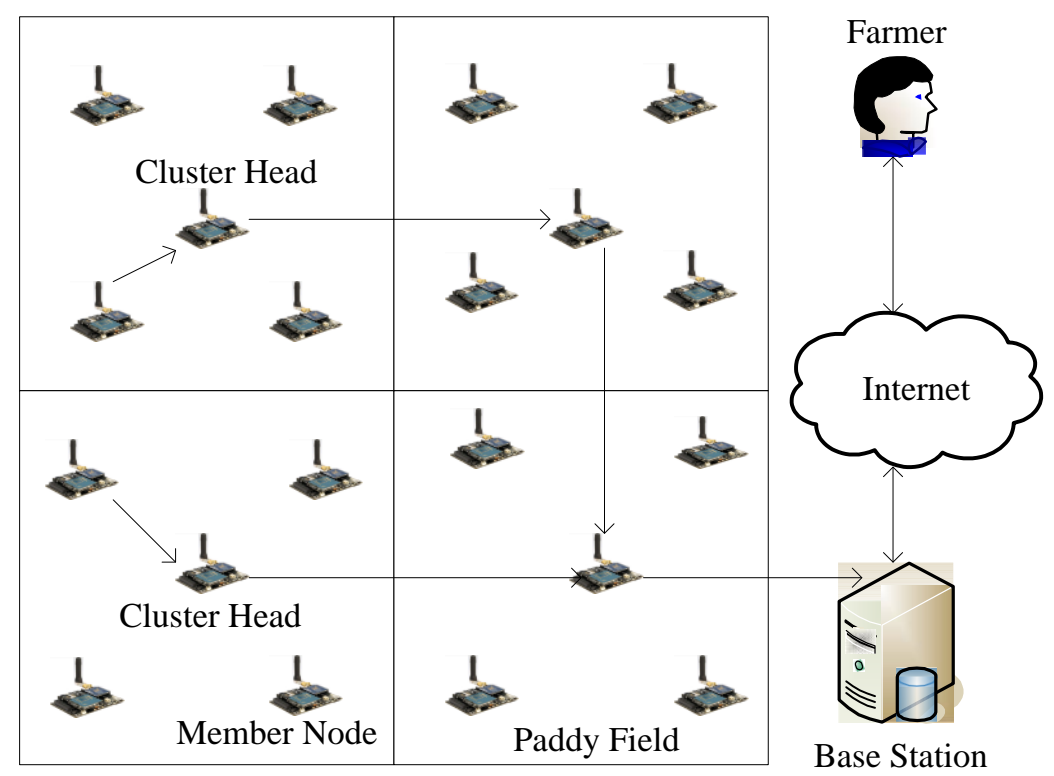

Figure 2. Paddy growth monitoring systems. 
The work done by Kait et al. [6] proposes the design of a sensor-based paddy growth monitoring system for improving rice yield. The architecture of their proposed system is shown in the Figure 2. The authors consider cost effectiveness as well as durability of the monitoring systems at outdoor operations. Sensor nodes are deployed in the field to gather data (temperature, humidity, light, water level etc.) periodically and send them to the base station (BS) using multi-hop routing. The BS processes data locally and sends to the remote server for further processing and analysis. After data analysis, an advisory message is sent to notify farmers about the field conditions and to provide suggestions. In case of an abnormal event, the BS sends alert messages to farmers. Experts can create a knowledge base using the data collected by sensors for further use/analysis. However, this monitoring system is not scalable and cannot be reused in other applications since it does not consider the concept of emerging M2M communication networks where thousands of machine type communication devices (MTCs) including sensors are connected to cover a large geographical area. Moreover, no paddy growth monitoring system based on the M2M communication networks exist in the literature.

\subsection{Irrigation System}

Traditional irrigation systems in agriculture consider uniform water distribution in fields. However, water requirements in the fields vary based on the soil condition (moisture, temperature etc) and the availability of water in the fields. Thus, designing an adaptive or field specific irrigation system is greatly required to reduce the environmental impacts, improve productions and to get more efficiency. In the irrigation monitoring system proposed in [2], the Bluetooth wireless transmitters sense the in-field soil moisture, temperature etc and send to the BS, which makes the field and time specific irrigation decision based on the sensed data. In a BS, a computer is connected to the Internet. The irrigation control unit gets the decision or, control signal processed by the software resides in the BS. The irrigation control units send the position of the irrigation system (e.g., sprinkler) using GPS receiver to the BS for the real time monitoring. The BS sends again control signals to the irrigation control station to operate the sprinkler based on the water requirement of the fields. Many researchers are also working on the variable-rate, sensor-based irrigation systems. Mirander et al. [7] propose a distributed irrigation system based on the soil water measurements. Shock et al. [8] propose a sensor-based irrigation system, where the data logger of a node sends the soil moisture data to the central computer for processing and control. Wall and King [9] propose an automated field-specific irrigation system with a new design for the soil moisture sensors and sprinkler valve controller. However, these irrigation systems do not deal with monitoring the pollution of water (e.g., water of lakes or rivers) and again do not consider the concept of M2M communication networks.

The seventh largest river in China is Huaihe, which spans 50 cities and 5 provinces. About 18\% food/agricultural products were produced in these areas in 2004 [10]. However, this river is polluted greatly due to industrial wastage, over fertilization and pesticides. It is estimated that $70 \%$ of the polluted water is used in irrigation. Hence, it is very important to 


\section{Macrothink}

develop sensor-based irrigation system that would monitor the reasons of pollutions and take appropriate measures to protect the water pollution. Moreover, it is also required to monitor the entire growth process of crop because the temperature, humidity requirements vary in the different growth period. In [10], Yang et al. develop an intensive sensor-based irrigation monitoring system for agriculture. This system is scalable (nodes can join in the network when require) and self- organizing (nodes and networks).

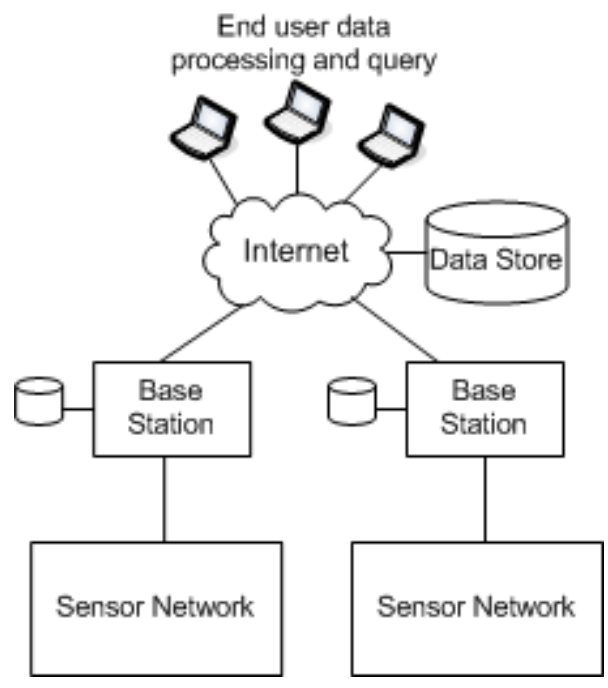

Figure 3. Bottom layer of irrigation monitoring system.

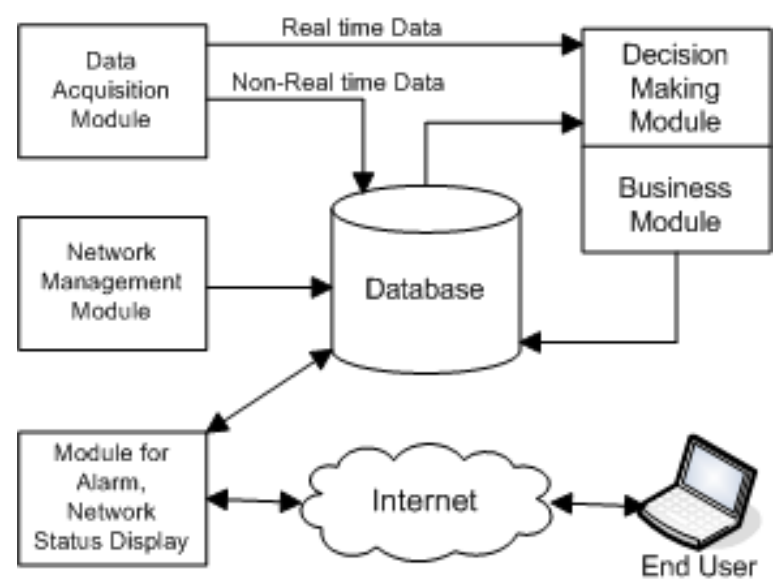

Figure 4. Upper layer of irrigation monitoring system.

The system architecture of their proposed sensor-based irrigation monitoring system [10] is divided into two layers: bottom layer and upper layer (Figures 3 and 4). Bottom layer consists of a hierarchical sensor network, where the sensor nodes are placed in widely separated clusters. Nodes send data to the BS where, BS is connected to wireless LAN (WLAN) and has a data logger. Upper layer consists of five modules: acquisition module, network management module, alarm/network status display module and business module. 
The data gathering module collects real or non-real time data from sensor networks, which are stored into the database and also used by the decision making module to make decision/alert notifications. Alarm/network status display module is responsible for alert notifications or displaying information to the end users. This module works as an access point between the end users and all other modules/networks. Network management module monitors the condition of the networks such as localization, collision and configures the network accordingly. The status information of the network is stored into the database by this module. The work done in [11] introduces automatic irrigation control based on M2M communication networks that conserve water and energy by using sensor intelligent network and efficient routing protocol. However, no significant amounts of works have been done in irrigation systems based on M2M communication networks.

\subsection{Precision Agriculture}

Precision agriculture (PA) is mainly used in developing countries. In PA, the differences of the agriculture related parameters such as temperature, humidity, soil moisture, rainfall among different fields are considered and analyzed to efficiently (precisely) utilize water, pesticides, fertilizer, seeds etc in fields. Hence, it becomes possible to increase the profit, reduce waste and maintain quality products. Though, GIS and GPS are used for PA, they are costly. Sensor networks provide a low cost technological solution for PA, for instance, in field crop management $[12,13]$ where sensor networks monitor crop conditions/growth for a longer period of time, make decision remotely and evaluate the potential of new crops. Moreover, using the data collected by sensors, it is possible to create a database or knowledge base of in-fields crops. Graphical user interfaces are integrated with the sensor network in these monitoring systems.

The PA manages fields differently based on the level of water, saline, field structure etc. The maps of the fields help to manage the fields and design the topology of sensor network (e.g., grid architecture). In this connection, different field management zones can be defined based on the temperature, humidity, water availability, sand quantity etc.

Agro-food productions still lacks of the usage of wireless technology, technical skilled persons due to high cost. Hence, PA is difficult to implement in agro-food [12]. Due to the low cost sensor nodes, the scalability and flexibility of sensor networks makes it popular in PA. Moreover, "Ambient Intelligence (AmI)" paradigm can further extend sensor networks applicability in PA. The work done by Chiti et al. proposes "next generation farm" [12] which uses AmI and sensor networks and provides feedback and adaptability and thus, increases productions in agro-food. They also propose a dynamic flooding routing protocol of sensor network, which outperforms the static flooding routing protocol. In dynamic flooding protocol, each node knows the position and remaining energy of their neighboring nodes. Each node sends data through the highest residual energy neighboring nodes. The proposed protocol and framework is implemented in the EU GoodFood Project [12]. On a trial basis, authors implemented AmI in a farm of the University of Florence, Italy and monitored a wine chain starting from the wine production to aging chain. Using a sensor network, they monitor leaf temperature, stem growth, diseases, fragmentation, ageing etc of a vineyard. They use 
MICA2 sensor nodes [12], which consist of up to 16 sensors and also use built-in routing protocol. Data collected by these sensors are routed to the gateway. Gateways further route the data to remote computers/servers for processing and analysis.

\subsection{Field Assessment}

Farmers require the state of the crop field to decide about the irrigation, applying fertilizers and pesticides. Farmers mostly rely on their mental models for this purpose; however, mental models might not always be optimal. Wark et al. [14] propose sensor network based field management system for this purpose, which uses solar powered moisture nodes and low resolution camera. Hence, information from two different sources is available for comparison, assessment and analysis. Self contained, self-powered low resolution camera nodes send the height, coverage and greenness information of the field grass through sensor networks to the BS. Then the BS allocates time for these camera nodes to send images. The camera nodes can also be used to observe cattle position and behavior.

\subsection{Cattle Monitoring and Behavior Control}

Monitoring the behavior of animals or cattle that is, how they interact each other and move in the field are very important to properly utilize the limited field resources (e.g. grass). For this purpose, Wark et al. propose sensor network based cattle monitoring system [14] where Fleck-2 cattle nodes (sensors) are placed in a plastic box before putting into the collars that the animals wear into neck. GPS, two batteries and radio antenna are also placed in the collar. The design of the collar is very important so that animals cannot damage it easily. Authors propose placing the radio frequency antenna flat on top of the collar. Zebranet [15] is a related project to monitor zebras using GPS positioning data.

Controlling animals is a challenge in the agricultural systems because their positional and mental state (e.g., stress, desire, mood) are very difficult to measure. Moreover, their behavior depends on many factors such as age, season, food availability, temperature etc. Sensor-based M2M networks can play an important role in behavior measure/control. In [14], Wark et al. focus on modeling cattle behavior based on their position and inertial data. For this purpose, adjusting the inertial sensors is very important to get useful information. A sensor calibration model is developed based on the distribution of inertial data. For instance, during day time, the activities of cattle are a lot more than that of during night. Hence, their grazing, ruminating, sleeping (behavioral) data will have different shape in different time (e.g., day and night). Calibration model is based on mapping these dataset to different parameters. From inertial sensors, data about the speed, turning rate and movement energy of cattle can be collected. These data help identifying cattle behavior. Wark et al. [14] also propose to integrate radio frequency identification tag (RFID) with sensor networks to record/track the amount of food each animal takes and send this information through the networks.

\subsection{Other Agriculture Monitoring Systems}

The work done by Yoo et al. [16] proposes a precision and intelligence agricultural system namely automated agriculture system $\left(\mathrm{A}^{2} \mathrm{~S}\right)$ based on sensor networks. The system 
consists of a number of wireless sensors to monitor and control of the agriculture fields (environment) and a management sub-system which controls sensor network and provides services to the farmers through PDA. They implemented $\mathrm{A}^{2} \mathrm{~S}$ to monitor and control the growing process of melon and cabbage in a greenhouse. Sensor networks have three types of nodes: twenty five A type sensor nodes, one $\mathrm{C}$ type actuator node and three sink nodes. All nodes are placed in a greenhouse except one which is placed in a field outside. Three gateways are used to transfer data from the sink node to the server. Here, WLAN is used to provide a long distance communication between sensor network and server. A management subsystem consists of a database, application and web server. The application server receives data from sensors and stores to the database server. Users (farmers) can access data through the web server/interface using PDA.

A-nodes comprise a 8 bit micro controller unit, transceiver of range $2.4 \mathrm{GHz}$, timer and sensors (for light, temperature and humidity). Each node also has application software, which reads the sensed attributes (temperature, humidity etc) of the sensors and sends to the sink node. The application software turns off the sensors of a node after getting the "sleep order message" from the sink. The node uses its timer to wake up the sensor again. C-nodes (actuator) have an additional relay board to control (turn on/off) the switch of the lights of greenhouse. Sink nodes do not have any sensor. These nodes receive the working schedule of the sensor nodes from the server and also send the received sensed data to the server. The nodes are organized in a parent-child tree structure. A node can join in the network by broadcasting a "parent" search packet including cost function (hop count). Only the nodes, which are one level up in the tree than that of the searching parent node can be the parent of the node by unicasting an "invite packet" to that node.

Among other agriculture monitoring systems, the green supply chain management of agriculture products [17] are becoming popular for "green, safety and healthy" [9] life around the world. However, there is a gap between the applied research in M2M communication networks and its applicability in many application domains including green supply chain management. Thus, an M2M framework has been introduced in [10] for green supply traceability system. This framework includes RFID, sensors, and smart phone to cooperatively work with neighboring nodes through a unique addressing scheme. However, this framework does not include any network management components such as routing, scheduling for sensors, and RFID network. Image processing for vineyard monitoring [18] and drone technology in agriculture [19] have achieved significant attention in recent years. The drone technology uses unmanned aerial vehicles (UAVs) for applying pesticides in vineyards and agriculture fields that are not easily accessible by human beings. Table 1 presents comparison among several existing sensor-based agricultural monitoring systems that we present in the literature. 
Table 1. Topology and routing protocols of sensor networks in agriculture monitoring systems.

\begin{tabular}{|c|c|c|c|c|c|}
\hline $\begin{array}{ll}\text { WSN-based } & \text { Agriculture } \\
\text { System } & \\
\end{array}$ & Region & Topology & Routing & $\begin{array}{l}\text { Energy } \\
\text { Efficient }\end{array}$ & $\begin{array}{l}\text { Fault } \\
\text { Tolerant }\end{array}$ \\
\hline $\begin{array}{l}\text { Paddy Growth Monitoring } \\
\text { System }\end{array}$ & Developed & Zone & Multi-hop & $\sqrt{ }$ & $\mathrm{X}$ \\
\hline $\begin{array}{l}\text { Village eScience for Life } \\
\text { (VESEL) }\end{array}$ & Developing & Zone & $\begin{array}{l}\text { Storage-Aware } \\
\text { Multi-Sink (multi-hop) }\end{array}$ & $\sqrt{ }$ & $\sqrt{ }$ \\
\hline COMMONSense Net (CNS) & Developing & Tree & Multi-hop & $\sqrt{ }$ & $\mathrm{X}$ \\
\hline $\begin{array}{ll}\text { Automated } & \text { Agriculture } \\
\text { System }\left(\mathrm{A}^{2} \mathrm{~S}\right) & \\
\end{array}$ & Developed & Tree & Multi-hop & $\sqrt{ }$ & $\mathrm{X}$ \\
\hline Next Generation Firm & Developed & Zone & $\begin{array}{ll}\text { Dynamic } & \text { Flooding } \\
\text { (Multi-hop) } & \end{array}$ & $\sqrt{ }$ & $\mathrm{X}$ \\
\hline $\begin{array}{l}\text { WSN-based } \\
\text { Systems }\end{array}$ & Developed & Tree & Local (Multi-hop) & $\mathrm{X}$ & $\sqrt{ }$ \\
\hline $\begin{array}{l}\text { WSN-based } \\
\text { Monitoring }\end{array}$ & Developed & Zone & Multi-hop & $\mathrm{X}$ & $\mathrm{X}$ \\
\hline
\end{tabular}

\subsection{Discussion}

Major challenges of the agricultural monitoring systems such as paddy growth monitoring systems [20] are power management and network lifetime. Batteries loose power over time and require recharging or replacement. Therefore, power consumption must be limited to the bare minimum required for effective performance. Research in this area focus on minimizing power consumption by limiting idle listening or reducing power loss during transmission through efficient routing. Managing the sleeping mode of sensor nodes to enable quick re-activation and appropriate sleep timing are very important. However, these problems can be resolved by using solar panels in agriculture fields to recharge the battery and upgrading the power class and antennas to increase the radio range. Existing agricultural monitoring systems are mostly suitable for a small area. In case of a large agriculture area, scalability and energy usage are critical for these frameworks. Moreover, the tree topology used in sensor-based intensive irrigation system [2] and other agriculture systems is not reliable because there is only one path from each node to the base station. Any link failure or node failure can make other nodes unreachable to the base station.

It is also very important to determine which wireless technology is more suitable for a sensor-based monitoring system to communicate between sensors and base station. Wi-Fi is a short range wireless technology and is used in mobile devices, e.g., PDA, laptop. Bluetooth is a high speed, short range and high data rate but low battery life wireless technology that replaces wired devices. Low rate wireless personal area network (LR-WPAN) is a low cost, low power and low data rate wireless technology used for inexpensive, fixed or portable mobile devices. In [21], authors choose WPAN in their proposed paddy growth monitoring system due to its low power consumption and long network lifetime. Bluetooth wireless technology is used in irrigation system presented in [2]. Automated agriculture system $\left(\mathrm{A}^{2} \mathrm{~S}\right)$ 
[16] uses WLAN to communicate between sensor networks and the central server. However, M2M communication networks are more suitable in agricultural monitoring systems since it comprises a large number of heterogeneous MTC devices and wireless technologies and supports mobility. Thus, M2M networks seem more optimal than the existing routing in cattle monitoring systems [14]. In the next section, challenges of sensor-based agriculture monitoring systems in developing countries are presented with technical requirements.

\section{Sensor-based Agriculture Monitoring Systems in Developing Countries}

A usable, adaptive and sustainable sensor-based M2M agriculture/field monitoring system is required for developing countries. This monitoring system should integrate a decision support system to optimize resource utilization, maximize production, and minimize environmental hazards. Moreover, this system should be autonomous, accurate, affordable (by poor farmers) and remotely controllable. The lifetime of this M2M monitoring system should be at least one crop season (4 -6 months) in developing countries. Though sensor-based monitoring systems have got a high potential in agriculture in developing countries, only a few projects have been implemented in developing regions, which are still in exploratory, iteration or evaluation phase.

\subsection{Design Challenges in Developing Countries}

As sensor networks are application specific, designing a sensor network for each application tends to the problem of limited resources and cost. Designing sensor-based M2M communication framework to use in multiple applications can eliminate these problems. On the other hand, sensor-based agriculture systems used in the precision agriculture of developed countries are not suitable in developing countries where almost $87 \%$ farmers are labor dependent and owe a small land. Economic conditions and information required by the farmers in developing countries are also different. Thus, the existing sensor-based M2M agriculture monitoring systems are required to be modified in this context.

The major design challenges of agricultural monitoring systems in developing countries are as follows [5, 19, 22].

- Cost - is the most important factor in the context of developing countries. Cost can be reduced by using sensor-based $\mathrm{M} 2 \mathrm{M}$ communication networks which are not currently being used in agricultural monitoring systems. Moreover, this single framework of M2M communication network can be used in multiple applications and also in precision agriculture which require densely deployed sensor nodes.

- Reliability - in most developing countries, sensor nodes are scarce and climates are extreme. Hence, sensor nodes must be covered to protect from outdoor conditions including moisture and heat. Availability and reliability of telecommunications/wireless infrastructure in rural areas of developing countries is another challenge. 


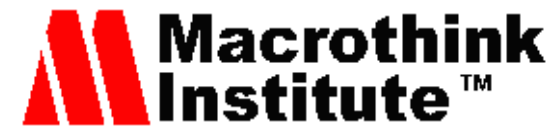

- Limited number of node deployment restricts a novel sensor network management scheme that uses alternate node scheduling. It also limits the usage of a power aware multi-hop routing that requires more sensor nodes.

- Developing a capable and intelligent information system for developing regions is very important. Otherwise, collecting only raw sensor data would be useless.

- Resource-poor farmers mostly rely on rain fed farming. Moreover, traditional irrigation is not efficient in semiarid areas in developing countries. Hence, a sensor-based M2M irrigation systems with a decision support system is required to maximize the effects of irrigation while minimize the intake of water.

- Crop models - simulate the growth of crops in different environmental constraints and play an important rule in designing decision support systems. Two existing crop models that are used in agricultural projects are DSSAT and APSIM [21]. These models consider only the crop growth and yields but do not consider the impact of pests and diseases and the timing of farming operations. Moreover, these models do not consider the recent development of environmental monitoring such as monitoring soil moisture. In such cases, data gathered from sensor-based MTC devices help to build, design and test more effective crop models. In addition, as it is very important to be roughly correct rather than precisely wrong, more environmental factors should be considered in the decision support systems/crop models that the farmers of developing regions require.

- The failures of information systems projects in developing countries are mainly due to the gap between the design and actual requirements, that is, the distances between the designers and users. Any participatory design does not guarantee any success until it alleviates the gap between the designers and users.

- Another major challenge in technology based agriculture systems in developing countries is the capability of users (farmers) to understand, use and own the system. The COMMONSense Net (CNS) project [21] provides a solution by using autonomous sensor network and apprenticeship to learn the system.

The CNS [14] is a sensor-based agriculture monitoring project developed for semiarid regions in India. CNS uses a participatory iterative design and implements a technology based solution in a very first iteration, uses and evaluates it in the local community/culture and performs improvements or redesign in the next iteration based on the feedback or output of the current iteration. Village eScience for Life (VESEL) [5] is another sensor-based agriculture project being implemented in developing regions in Africa. In the next subsection we present the architecture of CNS and VESEL projects.

\subsection{Technical Frameworks of Sensor-based Monitoring Systems}

In this section, we present technical framework especially, the topology and routing protocol used in CSN, VESEL projects and other agriculture monitoring systems for developing countries $[1,5,19]$. 


\subsubsection{Topology}

As agriculture fields in developing countries are homogeneous in terms of soil type, structure and crop type, a zone-based topology can be used, which requires less number of sensor-based MTC devices. However, the zone-based topology suffers from network coverage problem and reliability (i.e., how the system works when a node in a zone fails). In [5], Kabashi and Elmirghani propose a dynamic zone-based topology for the VESEL project. Initially, nodes are distributed to the zones based on the condition that each node remains within the range of the nodes of at least two zones. This overcomes the problem of node isolation. Then, each node identifies their neighboring nodes by broadcasting node/zone ID that is assigned by the gateway/coordinator nodes. Each zone node elects nodes in the neighboring zones to which they can connect with a minimum transceiver power. This generates several connected graphs, and the graph that requires minimum transmission power is selected for routing. Figure 5 presents the system architecture of VESEL project. The topology of sensor-based irrigation system described in [10] is a tree structure (single sink, multi source), where the only base station (BS) is at the root. However, this topology is not reliable since there is only one path from each node to the BS. Any link or node failure can make other nodes unreachable to the BS. The topology used for CNS project [21] is also tree structure and suffers from the link failure problem.

\subsubsection{Routing}

Kabashi and Elmirghani [5] propose a delay tolerant multi-sink architecture (Figure 5) and back-link/storage aware routing protocol for the VESEL project. Multiple sinks provide more storage capabilities and distributed data collection. Data are aggregated to several sink nodes. Sinks are located at the edge of a field and thus, supports automatic data logging when some hand-held community devices such as PDA (with wireless communication capabilities) pass by the sinks. In back-link/storage aware routing protocol, sinks and other nodes broadcast available memory/storage capacity. If the back link is available and of good quality, multi-hop routing protocol to find the next hop is used. Otherwise, if the storage capacity of sink is not enough, data will be stored locally or in neighboring nodes. In this way, routing balances or optimizes memory usage. 


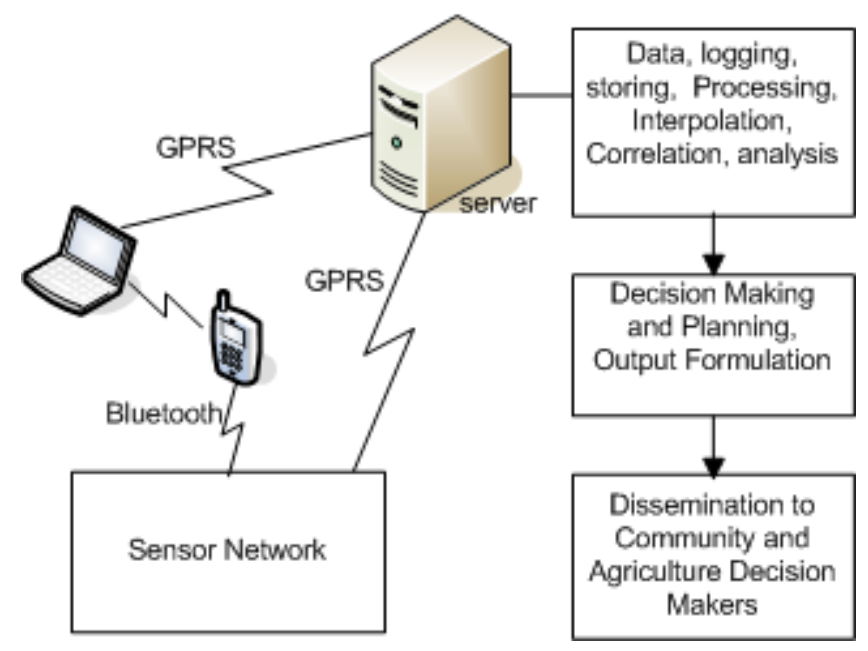

Figure 5. System architecture of VESEL project.

For routing in the tree topology of sensor-based irrigation systems [10], each node has an ID and neighboring node information table. Each node uses local routing, that is, broadcasts only data/packets to the neighbors. The BS initiates routing by sending a message of type 1 . Whenever, a node receives this message it keeps the neighbor information (from where it receives the message) into its routing table. Each node clears (except the BS) it routing table after $T$ cycles, when a new round will be initiated. Whenever a node wants to join the network or the routing information table of a node is empty, the node sends a joining request message of type 2 . The nodes, which are in the network and receive the type 2 message, reply with a type 3 message. The original node then compares the hop count of the nodes that sent the message of type 3 . If the hop count of that node is smaller than that of the original node, the node that replied the type 3 message can be a potential parent of the original node. The type 4 message from a child to parent is sent to know whether the parent is still alive. The parent replies by sending a type 5 message and the child updates its local routing table for its parent. If a child does not receive the type 5 message before a timer expires, the child assumes that the parent is dead and so, re-selects a parent. Re-selecting a parent may slow down the network. Moreover, any link failure may cause the BS unreachable.

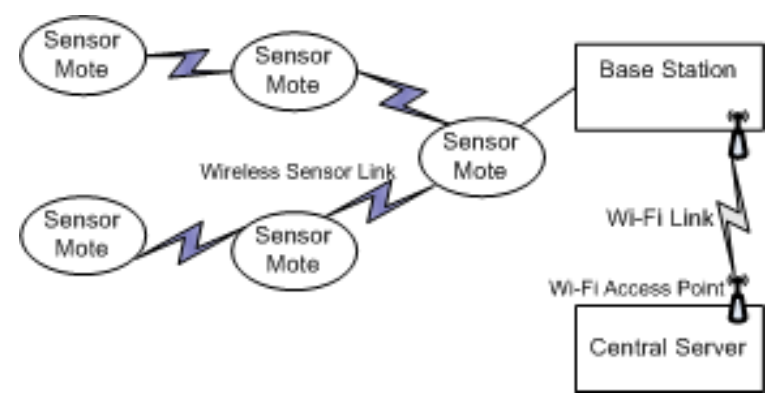

Figure 6. System architecture of COMMONSense Net. 


\subsubsection{Task Scheduling}

A dynamic task scheduling is proposed in the VESEL project. This scheduling considers the parameters to be sensed, energy of the resources and environmental factors (that affect sensing and network operations). This mechanism effectively schedules three system parameters: bit resolution, sampling rate and frequency of transmission. Initially, values of all these parameters are assigned/scheduled and then, adaptively rescheduled by a trigger from another environmental sensor (as agriculture parameters are inter-dependent). For instance, the soil moisture sensors can be triggered by rainfall or humidity sensors. Another way to refine/adjust the parameters is based on the profile of the parameters. System parameters also depend on the available node energy. For instance, when the energy is crucial, the bit resolution can be reduced to save the energy in analog-to-digital converter and conversion process. Scheduling is done at application layer by external server and coordinator nodes.

Table 2. Differences of agricultural monitoring systems in developed and developing countries

\begin{tabular}{|l|c|c|}
\hline \multicolumn{1}{|c|}{ Features } & $\begin{array}{c}\text { Developed } \\
\text { Countries }\end{array}$ & $\begin{array}{c}\text { Developing } \\
\text { Countries }\end{array}$ \\
\hline Extensive work on ICT-based agriculture & $\sqrt{ }$ & $\mathrm{X}$ \\
\hline Precision agriculture & $\sqrt{ }$ & $\sqrt{ }$ \\
\hline Scarce and wide sensor networks & $\sqrt{ }$ \\
\hline Dense and narrow sensor networks & $\mathrm{X}$ & $\sqrt{ }$ \\
\hline Delay Tolerance Network (DTN) architecture & $\sqrt{ }$ \\
\hline $\begin{array}{l}\text { Possibility of use of other technologies e.g. GPS, GIS, } \\
\text { remote sensing }\end{array}$ & $\sqrt{ }$ & $\mathrm{X}$ \\
\hline Requirement of real-time monitoring and accuracy & $\sqrt{ }$ \\
\hline Farmers are expert and able to operate the systems & $\sqrt{ }$ & $\mathrm{X}$ \\
\hline $\begin{array}{l}\text { Availability of a good telecommunication and wireless } \\
\text { infrastructure }\end{array}$ & & $\mathrm{X}$ \\
\hline
\end{tabular}

Figure 6 shows the systems architecture of CNS project. It uses a centralized data collection subsystem, where each node sends the collected data to the central sever through BS. As the nodes are not mobile and topology does not change frequently, CNS uses tree construction multi-hop routing algorithm. In sensing subsystem, Mica2 motes (includes temperature, humidity, ambient light sensors) are used. Mica2 nodes have low power consumptions and highest radio range among the current sensor technology. CNS uses multi-hop routing protocol that is embedded in the TinyOS software system. For saving energy, transmission rate is maintained lower than the sampling rate. For connecting different subsystems, CNS uses IEEE 802.11 (Wi-Fi) wireless links. A Java based application is used to $\log$ data and metadata in the database of sensor network. As there was no GSM/GPRS connectivity in rural areas when the CNS project was first implemented, expensive and energy inefficient Wi-Fi links/bridges were used. However, due to the recent improvement/connectivity of GPRS technology, it is being used in the CNS project as a bridge to aggregate and transmit data to the central server. 


\section{$\Lambda$ Macrothink}

\subsection{Proposed Sensor-based M2M Agricultural Monitoring System}

Based on the design challenges and existing technical framework of the agriculture monitoring systems we identify and present differences in sensor-based agriculture monitoring systems of developed and developing countries in Table 1. Considering all these challenges and technical frameworks, we propose a novel agriculture monitoring systems (with improved topology and routing protocol) for farmers in developing countries.

The proposed framework, as is illustrated in Figure 7 uses M2M communication networks by integrating sensors in agricultural field with other wireless devices such as PDA, smart phones (i.e., MTC devices that people use in their everyday life). Data collected from sensors are transmitted to central server through different wireless devices and networks (e.g., Wi-Fi, GPRS). The central server has different components for data storage, processing and notifications such as database management systems, alarm systems. The proposed framework also integrates an efficient crop model/decision support system that is suitable for agriculture in developing regions as well as building a timely and cost effective IT infrastructure through ICT apprenticeship as an iterative process. Besides, the framework focus on designing an energy efficient and reliable zone-based topology and routing protocol for agricultural sensor network where multiple base stations (BS) or gateway node can be placed outside of the network area so that sensors can communicate with the nearest BS to reduce energy consumptions. Moreover, a few more alternative sensor nodes can be distributed to zones that are closest to BS than those of the farthest zones from BS. An alternate becomes an active node after a fixed number of data sensing operations either if the current active node fails or is at energy critical condition. Efficient shortest path establishment to achieve energy efficiency, providing fault tolerance to achieve reliability of the framework should also be considered.

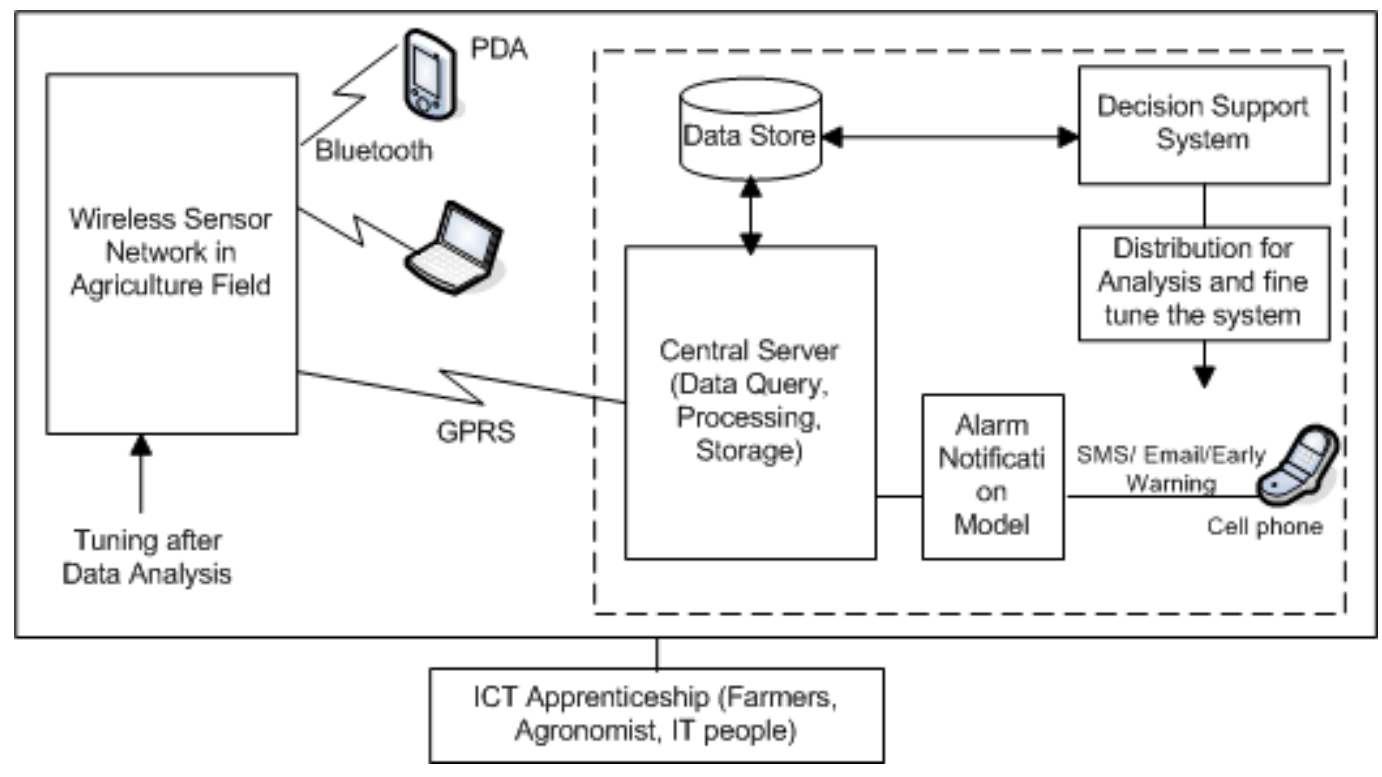

Figure 7. Proposed sensor-based M2M agriculture monitoring system 


\section{Summary}

This paper presents several existing sensor-based M2M agriculture monitoring systems with their working principles. Most of these monitoring systems are implemented for farmers in developed countries. However, a few iterative, participatory sensor-based agriculture monitoring systems are being designed and implemented in developing countries. As the information requirements of the farmers and socio-economic conditions in developing countries are different than those in developed countries, the agriculture monitoring systems for developing countries have different design requirements and technological frameworks. We also presented the key features on which these monitoring systems differ in developed and developing countries. Finally, we came up with the design and implementation of a novel sensor-based M2M agriculture monitoring system for developing countries. As a future work, we would study, classify and compare M2M-based agriculture monitoring systems.

\section{Reference}

[1] Edordu C. and Sacks L., "Self Organizing Wireless Sensor Networks as a Land Management Tool in Developing Countries: A Preliminary Survey," In Proceedings of the 2006 London Communications Symposium, September 2006, Communications Engineering Doctorate Centre, London, UK.

[2] Kim Y., Evans R.G. and Iversen W.M., "Remote Sensing and Control of an Irrigation System Using a Distributed Wireless Sensor Network," Instrumentation and Measurement, IEEE Transactions on, vol.57, no.7, pp.1379-1387, July 2008. http://dx.doi.org/10.1109/TIM.2008.917198

[3] Carles Antón-Haro, Thierry Lestable, Yonghua Lin, Navid Nikaein, Thomas Watteyne, Jesus Alonso-Zarate, "Machine-to-machine: An emerging communication paradigm. Trans. on Emerging Telecommunications Technologies", Volume 24, Issue 4, pages 353-354, June 2013. DOI: http://dx.doi.org/10.1002/ett.2668

[4] Moummadi, K., Abidar, R., Medromi, H., "Generic model based on constraint programming and multi-agent system for M2M services and agricultural decision support," Multimedia Computing and Systems (ICMCS), 2011 International Conference on, pp.1-6, Morocco, 7-9 April 2011. DOI: http://dx.doi.org/10.1109/ICMCS.2011.5945678

[5] Kabashi A.H. and Elmirghani J.M.H, "A technical framework for designing Wireless Sensor Networks for Agricultural Monitoring in Developing Countries", International Conference on Next Generation Mobile Applications, Services and Technologies (NGMAST), Cardiff, Wales, UK, Sept 2008.

[6] Xing G., Lu C., Pless R., and Huang Q., "Impact of sensing coverage on greedy geographic routing algorithms". IEEE Transactions on Parallel and Distributed Systems, 17(4):348-360, April 2006. DOI: http://dx.doi.org/10.1109/TPDS.2006.48

[7] Shock C. C., David R. J., Shock C. A., and Kimberling C. A., "Innovative, automatic, 
low-cost reading of watermark soil moisture sensors", in Proc. Irrig. Assoc. Tech. Conf., Falls Church, VA, 1999, pp. 147-152.

[8] Miranda F.R., Yoder R., and Wilkerson J.B., "A site-specific irrigation control system", presented at the ASAE Annu. Int. Meeting, Las Vegas, NV, Jul. 27-30, 2003. doi: http://dx.doi.org/10.13031/2013.13740

[9] Wall R.W. and King B.A., "Incorporating plug and play technology into measurement and control systems for irrigation management", presented at the ASAE/CSAE Annu. Int. Meeting, Ottawa, ON, Canada, Aug. 2004.

[10] Yang W., Liusheng H., Junmin W. and Hongli X., "Wireless Sensor Networks for Intensive Irrigated Agriculture," Consumer Communications and Networking Conference, 2007. CCNC 2007. 4th IEEE, pp.197-201, Las Vegas, Nevada, Jan. 2007. http://dx.doi.org/10.1109/CCNC.2007.46

[11] Zhang F., "Research on water-saving irrigation automatic control system based on internet of things," Electric Information and Control Engineering (ICEICE), 2011 International Conference on, pp.2541-2544, Wuhan, China, 15-17 April 2011. http://dx.doi.org/10.1109/ICEICE.2011.5778297

[12] Chiti F., De Cristofaro A., Fantacci R., Tarchi D., Collodo G., Giorgett G. and Manes, A., "Energy efficient routing algorithms for application to agro-food wireless sensor networks," Communications, ICC 2005. 2005 IEEE International Conference on, vol.5, pp. 3063-3067 Vol. 5, Seoul, South Korea,16-20 May 2005. http://dx.doi.org/10.1109/ICC.2005.1494957

[13] Konstantinos K., Apostolos X., Panagiotis K. and George S., "Topology Optimization in Wireless Sensor Networks for Precision Agriculture Applications," Sensor Technologies and Applications, 2007. SensorComm 2007. International Conference on, pp.526-530, Valencia, Spain, 14-20 Oct. 2007. http://dx.doi.org/10.1109/SENSORCOMM.2007.101

[14] Wark T., Corke P., Sikka P., Klingbeil L., Ying Guo, Crossman, C., Valencia P. and Swain D.; Bishop-Hurley G., "Transforming Agriculture through Pervasive Wireless Sensor Networks," Pervasive Computing, IEEE , vol.6, no.2, pp.50-57, April-June 2007. http://dx.doi.org/10.1109/MPRV.2007.47

[15] Zhang P., Sadler C.M., Lyon S.A., and Martonosi M., "Hardware design experiences in ZebraNet". In Proceedings of the 2nd international Conference on Embedded Networked Sensor Systems. SenSys '04. Baltimore, MD, USA, November 03 - 05, 2004, pp. 227-238. http://dx.doi.org/10.1145/1031495.1031522

[16] Yoo S., Kim J., Kim T., Ahn S., Sung J. and Kim D., "A ${ }^{2} S$ : Automated Agriculture System based on WSN," Consumer Electronics, 2007. ISCE 2007. IEEE International Symposium on, pp.1-5, Dallas, Texas, USA, 20-23 June 2007. http://dx.doi.org/10.1109/ISCE.2007.4382216

[17] Lin L., "Application of the Internet of Thing in Green Agricultural Products Supply 


\section{Macrothink}

Chain Management," Intelligent Computation Technology and Automation (ICICTA), 2011 International Conference on, vol.1, pp.1022-1025, Shenzhen, China, 28-29 March 2011. http://dx.doi.org/10.1109/ICICTA.2011.256

[18] Jaime Lloret, Ignacio Bosch, Sandra Sendra and Arturo Serrano, A Wireless Sensor Network that use Image Processing for Vineyard Monitoring, Sensors, Vol. 11, Issue 6, pp. 6165-6196. June 2011. http://dx.doi.org/10.3390/s110606165

[19] Uses and Policy Considerations of Unmanned Aerial Systems/Drones in Agriculture, http://ofbf.org/uploads/Drones_in_Agriculture.pdf, Accessed on October 2013

[20] Kait L.K., Kai C.Z., Khoshdelniat R., Lim S.M., and Tat E.H., "Paddy Growth Monitoring with Wireless Sensor Networks," Intelligent and Advanced Systems, 2007. ICIAS 2007. International Conference on, pp.966-970, Kuala Lumpur, Malaysia, 25-28 Nov. 2007. http://dx.doi.org/10.1109/ICIAS.2007.4658529

[21] Jacques P., Seshagiri R., Prabhakar, T.V., Jean-Pierre H. and Jamadagni, H. S., "COMMONSense Net: A Wireless Sensor Network for Resource-Poor Agriculture in the Semiarid Areas of Developing Countries", Jour. of Information Technologies and International Development, vol 4, no. 1, pp. 51-67, 2007.

[22] Zennaro M., Bagula A., Pehrson B., "Wireless Sensor Networks: a great opportunity for researchers in Developing Countries", 2nd IFIP International Symposium on Information Technology in Developing Countries, Pretoria, Oct 2008.

\section{Copyright Disclaimer}

Copyright reserved by the author(s).

This article is an open-access article distributed under the terms and conditions of the Creative Commons Attribution license (http://creativecommons.org/licenses/by/3.0/). 